\title{
G Somatem \\ Identification of the Glucose Metabolism \\ Biomarkers of NASH: Weighted Gene Co-Expression Network Analysis
}

\section{Xingyuan Chen}

Department of Gastroenterology, The Second Affiliated Hospital of Guangzhou Medical University, Guangzhou

\section{Zhanhui Ye}

Department of Gastroenterology, The Second Affiliated Hospital of Guangzhou Medical University, Guangzhou

\section{Kequan Chen}

Department of Gastroenterology, The First Affiliated Hospital of Guangzhou Medical University, Guangzhou

\section{Jiahui Xu}

Department of Gastroenterology, The Second Affiliated Hospital of Guangzhou Medical University, Guangzhou

\section{Liangying Ye}

Department of Gastroenterology, The Second Affiliated Hospital of Guangzhou Medical University, Guangzhou

\section{Yufeng Luo}

Department of Gastroenterology, The Second Affiliated Hospital of Guangzhou Medical University, Guangzhou

\section{Yangzhi Xu}

Department of Gastroenterology, The Second Affiliated Hospital of Guangzhou Medical University, Guangzhou

\section{Shiming Liu}

Guangzhou Institute of Cardiovascular Disease, The Second Affiliated Hospital of Guangzhou Medical University, Guangzhou

Hui Yang ( $\square$ yanghui@gzhmu.edu.cn )

Department of Gastroenterology, The Second Affiliated Hospital of Guangzhou Medical University, Guangzhou

\section{Research Article}

Keywords: NASH, WGCNA, MANBAL, MYC, CASP4, CDK5RAP3, ZFP36 
Posted Date: January 11th, 2022

DOI: https://doi.org/10.21203/rs.3.rs-1166724/v1

License: (c) (i) This work is licensed under a Creative Commons Attribution 4.0 International License. Read Full License 


\section{Abstract \\ Background}

The genetic mechanism of glucose metabolism has not been elucidated in nonalcoholic steatohepatitis (NASH), and many genes are took part in glucose metabolism of NASH. In this study, we used the weighted gene co-expression network analysis (WGCNA) to find the key genes associated with glucose metabolism;

\section{Methods}

Data sets GSE96971 and GSE89632 from Gene Expression Omnibus (GEO) were analyzed by WGCNA. We screened the Hub gene from the GSE96971 dataset, and the selected Hub genes were verified by GSE89632 dataset. We then analyzed the dataset using the Gene Ontology (GO) term enrichment and the Kyoto Encyclopedia of Genome (KEGG) path analysis. Expression levels of the hub genes are assessed by qPCR analysis. The function of hub genes was verified by Nile Red staining and relative glucose consumption detection;

\section{Results}

The hub genes are mannosidase beta like (MANBAL), myc proto-oncogene protein (MYC), caspase 4 (CASP4), CDK5 regulatory subunit associated protein 3 (CDK5RAP3) and ZFP36 ring finger protein (ZFP36) in the datasets GSE96971 and the GSE89632. Further, these genes are mainly involved in the integral component of membrane and plasma membrane, the PI3K-AKT signaling pathway and the olfactory transduction according to the GO and KEGG results. These hub genes were significantly upregulated in the palmitic acid (PA) cell model and methionine-choline-deficient medium (MCD) cell model. After knocking out the hub genes in PA model and the MCD model of NASH, relative glucose consumption was increased and lipid deposition was reduced compared with the control group;

\section{Conclusions}

MANBAL, MYC, CASP4, CDK5RAP3 and ZFP36 are elevated and involved in the pathogenesis of NASH. Further research on these genes are warranted.

\section{Introduction}

Non-alcoholic steatohepatitis (NASH), which affects $3-5 \%$ of the world's population, is a serious chronic liver disease, making it a major public health concern [1]. In the case of insulin resistance, the ability of insulin to inhibit lipolysis of adipose tissue was weakened, and more fatty acids were transported to the liver to synthesize triglyceride, leading to lipid accumulation in the liver and promoting the occurrence and 
development of NASH [2 - 3]. However, genetic factors that associated with NASH for insulin resistance have not been established [4]. Therefore, it is necessary to further explore and study the hub genetic genes of glucose metabolism in NASH patients.

The traditional differential gene expression analysis is to compare the differences in gene expression under two conditions and identify specific genes related to the conditions. The WGCNA is more helpful than the traditional differential gene expression analysis to reveal the genes associated with NASH disease [5]. WGCNA can divide the genes tested in the samples into different modules, and then conduct correlation and cluster analysis on the obtained modules to understand the specific situation of the modules. WGCNA can also analyze the relationship between modules and clinical information to find out which modules are most relevant to clinical information. Therefore, WGCNA analysis has greater biological significance than traditional difference analysis. [6].

In this study, WGCNA was used to analyze the microarray of liver biopsies from NASH patients to investigate the key molecules involved in glucose metabolism. At the same time, we annotated, visualized and integrated discovery (DAVID) and functionally analyzed the candidate genes. Finally, qPCR, Nile Red staining and glucose uptake rate experiments were used to verify the expression level and related functions of the target genes.

\section{Materials And Methods}

Upon reasonable request, the corresponding author can provide data and codes that support the results of this research.

\section{Microarray data and data preprocessing}

The GSE96971 dataset tested with Illumina HumanHT-12 WG-DASL V4.0 R2 expression chip consisted of $18 \mathrm{NASH}$ samples and the corresponding clinical data (age, gender, insulin resistance and other clinical information). The GSE89632 dataset tested with Illumina HumanHT-12 WG-DASL V4.0 R2 expression chip consisted of 19 NASH samples and 24 healthy control samples. We downloaded the GSE96971 and the GSE89632 from the Gene Expression Omnibus (GEO) (https://www.ncbi.nlm.nih.gov/geo/). The GSE96971 dataset is used as a training set. Hub gene was verified using GSE89632. Next, probe level data were transformed into gene-level data and the probe that does not match the genetic symbol is excluded. Take the average expression value as the gene expression value if multiple probes corresponding to the same gene [7]. The top 5000 genes with the largest coefficient of variation were screened out from the samples of WGCNA analysis.

\section{WGCNA}

Construct the gene network by R package WGCNA according to the WGCNA process[6]. First, extract the first 5000 differential genes from the expression matrix of GSE96971 dataset to construct the WGCNA coexpression network. In WGCNA analysis, the weighted value of correlation coefficient was used to make 
the connections between genes in the network obey the scale-free network distribution, and the logarithm of node probability was at least 0.8 . Then, the correlation matrix was transformed into an adjacency matrix, and the topological overlap matrix (TOM) describing the correlation between genes was obtained according to the adjacency matrix [8]. The cluster tree was obtained by dynamic tree cutting method (mergeCutheight $=0.25$, minmoduleSize $=30$, tomType $=$ "unsigned"), and the result of the cluster tree was used for module detection. Finally, we calculated the module signature genes in each module and correlated these signature genes with the clinical information of the NASH sample to identify the modules that were clinically relevant to NASH. In a WGCNA, each gene had its gene significance (GS) and module membership (MM). In WGCNA, when the gene significance (GS) and module member (MM) of a gene are close to 1, the gene is considered to have a high positive correlation with NASH [9].

\section{Identification of the hub gene and Visualization}

First, in the module, the selection of pivot genes was based on $G S>0.4$ and $M M>0.6$ as hub genes. Then use DAVID (https://david.ncifcrf.gov/home.jsp, version 6.8) to KEGG and GO enrichment analysis, in order to understand the key modules of the biological functions.At the same time, the String website (http://string-db.org/) and Cytoscape software were used to conduct visual analysis of key module genes and hub gene analysis to find the target genes in the pathogenesis of NASH.

\section{Cell culture}

AML12 cells purchase culture collections from cell type banks (CBTCC, Chinese Academy of Sciences, Shanghai, China). The medium containing DMEM/F12 (Gibco, Carlsbar,CA) supplemented with 10\% FBS (BI's Fullback, Israel), $1 \%$ Insulin- transferrin-Selenium (Sciencell, USA), $0.2 \%$ dextramethasone (DM, SigmaAldrich $1 \%$ penicillin-streptocin solution (PS, Sigma-Aldrich, USA) was used to culture AML12 cells. AML12 cells were cultured at $5 \% \mathrm{CO} 2$ humidity at $37^{\circ} \mathrm{C}$.

\section{NASH cell model}

\section{MCD model}

AML12 cells were spreaded on the six-hole plates at the density of $1.5 \times 10^{5} /$ hole. Discard the medium in the hole after $24 \mathrm{~h}$. In order to starve cells, add $2 \mathrm{ml}$ of DMEM/F12 without FBS to each hole for 12 hours. After $12 \mathrm{~h}$, add $2 \mathrm{ml}$ MCD (Gibco, Carlsbad, CA) with 10\% FBS medium to each hole of the MCD model group, and add $2 \mathrm{ml}$ of DMEM/F12 with $10 \% \mathrm{FBS}, 1 \% \mathrm{ITS}, 0.2 \%$ DM and $1 \%$ PS to each hole of the normal control group as well. After $12 \mathrm{~h}$, suck the medium in each hole of MCD model group out and place it in a sterile $4 \mathrm{ml}$ centrifuge tube. Refill the hole after adding Lipopolysaccharide(LPS) to make the working concentration to $1 \mu \mathrm{g} / \mathrm{ml}$. Continue the culture for $12 \mathrm{~h}$.

\section{PA model}

AML12 cells were spreaded in the six-well plates at the density of $1.5 \times 10^{5} /$ well, and discard the medium after 24h.In order to starve cells, add DMEM/F12 without FBS to each well. After $12 \mathrm{~h}$, add $1 \mathrm{Mm}$ PA lipidmedium ( $50 \mathrm{ml} 1 \mathrm{Mm}$ PA lipid medium is make up of $6 \mathrm{ml} 2.13 \mathrm{mg} / \mathrm{ml} \mathrm{PA}, 5 \mathrm{ml} 220 \mathrm{mg} / \mathrm{ml}$ Bovineserum 
albumin (BSA) and $39 \mathrm{ml}$ DMEM/F12) to each hole of the PA model group for $24 \mathrm{~h}$, and add $2 \mathrm{ml}$ of DMEM/F12 with $10 \%$ FBS, $1 \%$ ITS, $0.2 \%$ DM and $1 \%$ PS to each hole of the normal control group as well.

\section{Expression Levels of the Centered Genes Assessed by Quantitative Real-time PCR Analysis}

AML12 cells were treated with MCD and PA to build NASH cell models. Subsequently, extract total RNA from per sample using (Trizol TaKaRa, Dalian, China). Next, depended on LightCycler ${ }^{\circledR} 480$ II real-time PCR system (Roche), perform quantitative real-time polymerase chain reaction (qRT-PCR) with SYBRß Premix Ex Taq ${ }^{\text {TM }}$ Kit (Takara) at the temperature of $95^{\circ} \mathrm{C}$ for 2 minutes, continued with 40 cycles with the temperature of $95^{\circ} \mathrm{C}$ for 30 seconds, $53^{\circ} \mathrm{C}$ for 30 seconds and $72^{\circ} \mathrm{C}$ for 30 seconds. Our results were normalized to the expression of glyeraldehyde-3-phosphate dehydrogenase (GAPDH). The specific primers used for qRT-PCR are listed in support Table 1. The relative expression of each hub gene between controls and MCD/PA models was determines by the $2-\triangle \Delta$ Ct method.

Table 1

Polymerase Chain Reaction Primers for Quantitative Real-Time Polymerase Chain Reaction

\begin{tabular}{|ll|}
\hline Gene & Primer sequence $\left(\mathbf{5}^{\prime} \mathbf{3}^{\prime} \mathbf{)}\right.$ \\
\hline MANBAL & F:ATCTTCCAGCTCATCTGTGTC \\
& R:TTCTTCTTCGTCTCCTTCTTCG \\
\hline CDK5RAP3 & F:CTCTGACTCTTCTGGAATACCC R:CTCTACTGCTCTCTGAGACAAG \\
\hline MYC & F:AAATCCTGTACCTCGTCCGATT \\
& R:CCACAGACACCACATCAATTTC \\
GAPDH & F:CATCACTGCCACCCAGAAGACTG \\
& R:ATGCCAGTGAGCTTCCCGTTCAG \\
\hline
\end{tabular}

The functions of the hub genes were verified by Nile Red staining and glucose consumption detection Nile Red staining

Take out the six-hole plates from the cell incubator, discard the medium in the plate, wash the cells with PBS twice, and extract PBS. Add $1 \mathrm{ml} 4 \%$ paraformaldehyde per hole to fix 30min, then discard $4 \%$ paraformaldehyde, and wash it with PBS twice. $1 \mathrm{ml} 1 \mu \mathrm{M}$ Nile Red staining solution was added to each hole and dyed away from light for $15 \mathrm{~min}$ at room temperature, then discarded Nile Red dye solution in the hole and washed with PBS three times. The nucleus was stained with DAPI cell staining solution without light at room temperature for $30 \mathrm{~min}$. The DAPI cell staining solution in the hole was discarded and washed with PBS for three times, each time is $1 \mathrm{~min}$. After the staining was completed, the fluorescence inverted microscope was used to take pictures. The magnification was 40 times, and the intracellular lipid deposition was observed by fluorescence imaging. 


\section{Glucose consumption detection}

Quantify the glucose concentration in liquid medium with an assay kit (E1010, Applygen Technologies, Beijing). Aspirate the growth medium and evaluate the glucose concentrationsof each treatment group according to the instructions of the manual. Use the BSA method to measure the protein concentration of cells in each group. Calculate glucose consumption (mM) as (level of glucose in the growth medium level of glucose in cell supernatant); while calculate relative consumption of glucose (mM) as (glucose consumption / protein concentration).

\section{Statistical Analysis}

Statistical analysis was performed with SPSS 20.0 (SPSS, USA) and statistical comparisons were performed with the Kruskal-Wallis test followed by all pairwise multiple comparisons. P-value $<0.05$ was considered statistically significant.

\section{Results}

\section{Microarray data Overview and Preprocessing}

We downloaded the raw data of GSE96971 (GSM2547018-GSM2547026) from GEO, which was further divided into cluster 1 (2 samples) and Cluster 2 (7 samples) (Fig. 1).

\section{Modules in the GSE96971 dataset}

Firstly, the correlation coefficient of 0.8 and the weight coefficient $(\beta)$ of 6 were selected as parameters to construct the co-expression network using WGCNA (Fig. 2A,B). According to the similarity between gene expressions, the hierarchical clustering was carried out to obtain the gene cluster tree. The tree was cleaved by dynamic cutting algorithm and the genes with consistent expression were divided into the same module. Lastly, 10 differential co-expression modules were generated (Figure 2C). We found that the grey module genes did not cluster into other nine modules.

\section{Modules analysis and identified}

To determine the co-expression similarity between NASH and ME, we calculated the Pearson correlation coefficient (PCC). Correlation among these parameters was subjected to Clustering (Figure 3A). Clustering results generates 9 modules, which were branched into two clusters. The results of adjacency similarity are shown by heat map (Figure 3B). Next, we analyzed the genes in the yellow-green module to explore the correlation between the genes in this module and insulin resistance (Figure 3C).

\section{Screening and verification of the hub gene}

We searched for the HUB gene in the yellow-green module of the GSE96971 dataset, and verified the hub gene found in the GSE89632 dataset (Figure 4). First, we screened the hub gene(GS>0.4 and MM>0.6) in 
yellow-green module. A total of 2,050 genes were identified as hub genes. In addition, we used GSE89632 data set to verify the selected HUB gene. GSE89632 dataset is divided into 10 modules via WGCNA method. The grey module cannot be clustered into other 9 modules (Figure $4 \mathrm{~A})$. The hub genes (GS>0.4 and $M M>0.6)$ in the insulin resistance module of GSE89632 were identified. The hub genes screened for the above two data sets were verified by visualization for Venn diagram (Figure 4B). The results of Venn diagram intersection showed that MANBAL, MYC, CASP4, CDK5RAP3 and ZFP36 were the hub genes shared by the two data sets. By STRING and Cytoscape analysis, MYC was found to be located in the center of the network formed by five hub genes (Figure 4C).

\section{GO functional enrichment analysis and KEGG pathway analysis}

We figure out the biological function of genes in the greenish-yellow module of GSE96971 data set via DAVID database. The top ten GO $(P<0.05)$ and KEGG $(P<0.05)$ terms of the greenish-yellow module were obtained (Fig. 5). The DAVID databases were utilized for gene enrichment analysis. GO analysis confers that these genes are integral component of membrane and plasma membrane, while KEGG pathway analysis further verified that these genes are involved in the olfactory transduction and $\mathrm{PI} 3 \mathrm{~K}$ AKT signaling pathway.

\section{Quantitative RT-PCR Analysis}

Since the role of Casp4 and ZFP36 in NASH has been reported, we only conducted further experimental verification studies on MANBAL, MYC and CDK5RAP3. The mRNA expression levels of MANBAL, MYC and CDK5RAP3 in two MCD and PA cells model were quantified via qRT-PCR. Compared with healthy controls, MANBAL, MYC and CDK5RAP3 mRNA levels were significantly up-regulated in NASH cell model group $(P<0.05)$ (Fig. 6).

\section{Roles of MANBAL, MYC and CDK5RAP3 in NASH}

To further confirm the functions of MANBAL, MYC and CDK5RAP3 in NASH, we also performed Nile Red staining assay and glucose consumption assay (Fig. 7). After the knockout of MANBAL, MYC and CDK5RAP3 in the NASH cell models, lipid depositions were significantly lessened and glucose uptake increased compared with simple NASH cell model groups (Fig. 7A, B).

\section{Discussion}

In this study, we downloaded the profile data from the GEO database. The expression profile data included clinical information about insulin resistance in NASH patients and then we analyzed the chip data using WGCNA. According to the correlation with NASH, a total of 10 modules were constructed in 
this study. Among the up-regulated modules, the greenyellow module had the strongest association with insulin resistance. The hub genes(MANBAL, MYC, CASP4, CDK5RAP3 and ZFP36)screened by GSE96971 and GSE89632 were mutually verified and displayed in the form of Venn Diagram. Through the analysis of STRING and Cytoscape, MYC is at the center of a network of five central genes by the 5 hub genes. Then, DAVID databases were used to conduct gene $\mathrm{GO}$ and KEGG analysis. These genes are mainly involved in the integral component of the membrane and the plasma membrane according to the Go results. The KEGG analysis shows that these genes are took part in the Olfactory transduction and PI3KAKT signaling pathway. Since the role of Casp4 and ZFP36 in NASH has been reported [10, 11], we only conducted further experimental verification studies on MANBAL, MYC and CDK5RAP3. Through further validation, MANBAL, MYC and CDK5RAP3 were significantly up-regulated in the PA model and MCD model. After gene knockout of MANBAL, MYC and CDK5RAP3 in NASH cell models, relative glucose consumption was increased and lipid deposition was reduced compared with the control group in the PA model and MCD model. Therefore, we believe that these three genes are related to lipid deposition and abnormal glucose metabolism in NASH, which may be a potential disease assessment marker and therapeutic target for NASH.

MANBAL is an 85 amino acid single-pass membrane protein belonging to the UPF0239 family that may have similar functions [12]. Diseases associated with MANBAL include colorectal cancer [13]. MANBAL targets proteins to lysosomes and endosomes through the mansweet-6-phosphate receptor and promoting disease processing [14]. The role of MANBAL in NASH has not been reported.

There are many reports on MYC expression and regulation function. Many studies suggest that MYC can interact with many transcription factors[15]. NF-kappa B is an important regulator of mice and human MYC promoters in B cells [16]. In addition to transcription initiation, MYC also plays a major role in regulating the transcriptional elongation of genes [17]. MYC has a potential role in glycolysis transformation, and increased expression of MYC during T cell mitosis increases glycolysis by a factor of 20 [18].

CDK5RAP3 is a protein-coding gene in the long arm of chromosome 20 [19]. CDK5RAP3 has a variety of transcriptional variants, forming different isoforms [20]. Diseases associated with CDK5RAP3 include renal cancer, hepatocellular carcinoma and diabetes [21,22,23]. It is reported that this protein plays an important role in signal pathways that control transcriptional regulation and cell cycle progression [24]. NF-KB signaling pathway is one of the pathways CDK5RAP1 targets in the apoptosis of human malignant melanoma A375 cells [25]. Concurrently, CDK5RAP3 regulates mitotic G2/M transition checkpoint and mitotic G2 DNA damage checkpoint [26]. CDK5RAP3 plays an apoptotic role through nuclear membrane rupture[27]. However, the role of CDK5RAP3 in NASH has also not been reported.

CASP4 is a protein-coding gene. CASP4 is involved in signal transduction pathways of apoptosis, necrosis and inflammation [28,29]. Diseases associated with CASP4 include Alzheimer's disease and nephropathic cystinosis $[30,31]$. New evidence suggests that activation of NLRP3 inflammasomes is an 
important regulator of inflammatory processes. NLRP3 plays a variety of roles in the development of NAFLD, through the induction of apoptosis and pyroptosis by the caspase family [32].

ZFP36 is a protein-coding gene. Diseases associated with ZFP36 include colorectal cancer and atherosclerotic lesions [33, 34]. ZFP36 plays a role in anti-inflammatory response [35]; ZFP36 inhibits tumor by stimulating ARE-mediated TNF-a mRNA decay and interferon and/or lipopolysaccharideinduced other ARE-containing inflammatory mRNAs in macrophages [36]. RNA sequencing have found increased expression of ZFPs in animal models of fatty liver [37]. ZNF can be used as a transcription cofactor to promote adipogenesis and acts as a splicing factor to promote the adipocyte differentiation [38][39].

\section{Conclusions}

In conclusion, MANBAL, MYC, CASP4, CDK5RAP3 and ZFP36 were identified as key hinge genes of NASH by WGCNA microarray data from human liver tissue. However, this study still has several limitations. First, the two microarray datas were from human liver tissue. However, experimental validations were conducted on NASH in vitro models. Further experiments will be required to verify these findings using liver tissues of NASH patients and NASH in vivo models. Second, hub genes screened from liver tissue expression profile did not be validated in peripheral samples before utilizing these genes as potential biomarkers. We have been collecting proper human samples to confirm this finding in our future study.

\section{Abbreviations}

$\mathrm{NASH} \quad$ Nonalcoholic steatohepatitis

WGCNA Weighted gene co-expression network analysis

GEO Gene Expression Omnibus

GO Gene Ontology

KEGG Kyoto Encyclopedia of Genes and Genomes

MANBAL Mannosidase beta like

MYC Myc proto-oncogene protein

CASP4 Caspase 4

CDK5RAP3 CDK5 regulatory subunit associated protein 3

ZFP36 ZFP36 ring finger protein

PA Palmitic acid 
MCD Methionine-choline-deficient medium

DAVID Visualization and integrated discovery

TOM Topological overlap matrix

MM Module membership

GS Gene significance

\section{Declarations}

\section{Ethics approval and consent to participate}

Not applicable.

\section{Consent for publication}

Not applicable.

\section{Availability of data and materials}

The datasets generated during and/or analyses during the current study are available in the Gene Expression Omnibus (GEO) repository. Dataset GSE96971 is stored at https://www.ncbi.nlm.nih.gov/geo/query/acc.cgi?acc=GSE96971) and dataset GSE89632 is stored at https://www.ncbi.nlm.nih.gov/geo/query/acc.cgi?acc=GSE89632). Both the datasets are freely available.

\section{Competing interests}

The authors declare that they have no competing interests.

\section{Funding}

This work was funded by the following grants: Key projects of universities of Guangdong Province Office of Education (NO. 2019KZDXM006 ), General Project of Guangdong Natural Science Foundation (NO. 2020A1515011255), Youth Fund Projects of Basic and Applied Basic Research of Guangdong Province NO. 2019A1515110081 and NO. 2019A1515110652 and Young Talents Program of Guangzhou Education Bureau (NO. 201831831).

\section{Authors' Contributions}

Conceived and designed the experiments: Xingyuan Chen, Zhanhui Ye, Kequan Chen and Hui Yang. Performed the experiments: Zhanhui Ye. Analyzed the data: Xingyuan Chen. Contributed 
reagents/materials/analysis tools: Shiming Liu, Hui Yang. Wrote the paper: Jiahui Xu, Liangying Ye, Yufeng Luo, Yangzhi Xu.

\section{Acknowledgments}

Not applicable.

\section{Supplementary Materials}

There are no supplementary materials.

\section{Author details}

1 Department of Gastroenterology, The Second Affiliated Hospital of Guangzhou Medical University, No.250 Changgang East Road, Haizhu District, Guangzhou, China. 2 Department of Gastroenterology, The First Affiliated Hospital of Guangzhou Medical University, NO.151 Yanjiang Road, Yuexiu District, Guangzhou, China. 3 Guangzhou Institute of Cardiovascular Disease, The Second Affiliated Hospital of Guangzhou Medical University, No.250 Changgang East Road, Haizhu District, Guangzhou, China.

\section{References}

1. Boeckmans J, Natale A, Rombaut M, Buyl K, Vanhaecke T, Rogiers V, Rodrigues RM, De Kock J. Flow cytometric quantification of neutral lipids in a human skin stem cell-derived model of NASH. MethodsX. 2020 Sep 19;7:101068.

2. Friedman S L, Neuschwander-Tetri B A, Mary R, et al. Mechanisms of NAFLD development and therapeutic strategies[J]. Nature Medicine, 2018.

3. Finck B N . Targeting Metabolism, Insulin Resistance, and Diabetes to Treat Nonalcoholic Steatohepatitis[J]. Diabetes, 2018, 67(12):2485-2493.

4. Dongiovanni P, Romeo S, Valenti L. Genetic Factors in the Pathogenesis of Nonalcoholic Fatty Liver and Steatohepatitis. Biomed Res Int. 2015;2015:460190.

5. Zhao W , Langfelder P, Fuller T, et al. Weighted Gene Coexpression Network Analysis: State of the Art[J]. Journal of Biopharmaceutical Statistics, 2010, 20(2):281-300.

6. Langfelder P, Horvath S. WGCNA: an R package for weighted correlation network analysis. BMC Bioinformatics. 2008 Dec 29;9:559.

7. Irizarry RA, Hobbs B, Collin F, Beazer-Barclay YD, Antonellis KJ, Scherf U, et al. Exploration, normalization, and summaries of high density oligonucleotide array probe level data. Biostatistics 2003;4(2):249-64.

8. Kogelman L J , Kadarmideen H N . Weighted Interaction SNP Hub (WISH) network method for building genetic networks for complex diseases and traits using whole genome genotype data[J]. BMC Systems Biology, 2014, 8(Suppl 2):S5. 
9. Xiao KW, Li JL, Zeng ZH, Liu ZB, Hou ZQ, Yan X, Cai L. Monocytes affect bone mineral density in preand postmenopausal women through ribonucleoprotein complex biogenesis by integrative bioinformatics analysis. Sci Rep. 2019 Nov 21;9(1):17290.

10. Aizawa S, Brar G, Tsukamoto H. Cell Death and Liver Disease. Gut Liver. 2020 Jan 15;14(1):20-29.

11. Hou C, Feng W, Wei S, Wang Y, Xu X, Wei J, Ma Z, Du Y, Guo J, He Y, Kong F, Tang R, Zheng K. Bioinformatics Analysis of Key Differentially Expressed Genes in Nonalcoholic Fatty Liver Disease Mice Models. Gene Expr. 2018 Dec 14;19(1):25-35.

12. Petushkova N A, Ivleva T S , Yam V . Human chorionic beta-mannosidase: comparison with betamannosidase from human cultured fibroblasts.[J]. Prenatal Diagnosis, 2010, 12(10):835-839.

13. La Rosa FA, Pierce JW, Sonenshein GE. Differential regulation of the c-myc oncogene promoter by the NF-kappa B rel family of transcription factors. Mol Cell Biol. 1994 Feb;14(2):1039-44.

14. Bush J , Temesvari L , Rodriguez-Paris J , et al. A role for a Rab4-like GTPase in endocytosis and in regulation of contractile vacuole structure and function in Dictyostelium discoideum.[J]. Molecular Biology of the Cell, 1996, 7(10):1623-1638.

15. Agarwal E , Altman B J , Seo J H , et al. Myc-mediated transcriptional regulation of the mitochondrial chaperone TRAP1 controls primary and metastatic tumor growth[J]. Journal of Biological Chemistry, 2019, 294.

16. Arsura M , Wu M , Sonenshein G E. TGF beta 1 inhibits NF-kappa B/Rel activity inducing apoptosis of B cells: transcriptional activation of I kappa B alpha.[J]. Immunity, 1996, 5(1):31.

17. Snyder RC, Miller DM. Regulation of c-myc transcription initiation and elongation. Crit Rev Oncog. 1992;3(3):283-91.

18. Kunkl M , Sambucci M , Ruggieri S , et al. CD28 Autonomous Signaling Up-Regulates C-Myc Expression and Promotes Glycolysis Enabling Inflammatory T Cell Responses in Multiple Sclerosis[J]. Cells, 2019, 8(6).

19. Mak GW, Chan MM, Leong VY, Lee JM, Yau TO, Ng IO, Ching YP. Overexpression of a novel activator of PAK4, the CDK5 kinase-associated protein CDK5RAP3, promotes hepatocellular carcinoma metastasis. Cancer Res. 2011 Apr 15;71(8):2949-58.

20. Kraemer N , Issa-Jahns L , Neubert G , et al. Novel Alternative Splice Variants of Mouse CDK5RAP3[J]. PLoS ONE, 2015, 10(8):e0136684.

21. Li J, Hu X, Su M, Shen H, Qiu W, Tian Y. CDK5RAP3 Participates in Autophagy Regulation and Is Downregulated in Renal Cancer. Dis Markers. 2019 Apr 2;2019:6171782.

22. Mak GW, Lai WL, Zhou Y, Li M, Ng IO, Ching YP. CDK5RAP3 is a novel repressor of p14ARF in hepatocellular carcinoma cells. PLoS One. 2012;7(7):e42210.

23. Liu KC, Leuckx G, Sakano D, Seymour PA, Mattsson CL, Rautio L, Staels W, Verdonck Y, Serup P, Kume $\mathrm{S}$, Heimberg $\mathrm{H}$, Andersson O. Inhibition of Cdk5 Promotes $\beta$-Cell Differentiation From Ductal Progenitors. Diabetes. 2018 Jan;67(1):58-70. 
24. Gerakis Y, Quintero M , Li H , et al. The UFMylation System in Proteostasis and Beyond[J]. Trends in Cell Biology, 2019, 29(12).

25. Xiong J, Wang Y, Gu Y, Xue Y, Dang L, Li Y. CDK5RAP1 targeting NF-KB signaling pathway in human malignant melanoma A375 cell apoptosis. Oncol Lett. 2018 Apr;15(4):4767-4774.

26. Jiang $\mathrm{H}$, Luo S , Li H . Cdk5 activator-binding protein C53 regulates apoptosis induced by genotoxic stress via modulating the G2/M DNA damage checkpoint.[J]. Journal of Biological Chemistry, 2005, 280(21):20651-20659.

27. Wang H, Wei L I, Changli L I, et al. CDK5RAP1 deficiency induces cell cycle arrest and apoptosis in human breast cancer cell line by the ROS/JNK signaling pathway[J]. Oncology Reports, 2015.

28. Valentín-Acevedo A, Sinquett FL, Covey LR. c-Rel deficiency increases caspase-4 expression and leads to ER stress and necrosis in EBV-transformed cells. PLoS One. 2011;6(10):e25467.

29. Bian Z M , Elner S G , Elner V M . Dual Involvement of Caspase-4 in Inflammatory and ER StressInduced Apoptotic Responses in Human Retinal Pigment Epithelial Cells[J]. Investigative Ophthalmology \& Visual Science, 2009, 50(12):6006.

30. Hitomi, J. Involvement of caspase-4 in endoplasmic reticulum stress-induced apoptosis and A $\beta-$ induced cell death[J]. The Journal of Cell Biology, 2004, 165(3):347-356.

31. Sansanwal P, Kambham N, Sarwal M M . Caspase-4 may play a role in loss of proximal tubules and renal injury in nephropathic cystinosis[J]. Pediatric Nephrology, 2010, 25(1):105-109.

32. Zhu W, Feng P P, He K, et al. Liraglutide protects non-alcoholic fatty liver disease via inhibiting NLRP3 inflammasome activation in a mouse model induced by high-fat diet[J]. Biochemical \& Biophysical Research Communications, 2018.

33. H. Zhang, W. R. Taylor, G. Joseph, et al. mRNA-Binding Protein ZFP36 Is Expressed in Atherosclerotic Lesions and Reduces Inflammation in Aortic Endothelial Cells[J]. Arteriosclerosis, Thrombosis, and Vascular Biology, 2013.

34. Montorsi L , Guizzetti F , Alecci C , et al. Loss of zfp36 expression in colorectal cancer correlates to wnt/ ß-catenin activity and enhances epithelial-to-mesenchymal transition through upregulation of zeb1, sox9 and macc1[J]. Oncotarget, 2016, 7(37):59144-59157.

35. Jin WJ, Chen CF, Liao HY, Gong LL, Yuan XH, Zhao BB, Zhang D, Feng X, Liu JJ, Wang Y, Chen GF, Yan HP, He YW. Downregulation of the AU-rich RNA-binding protein ZFP36 in chronic HBV patients: implications for anti-inflammatory therapy. PLoS One. 2012;7(3):e33356.

36. Aguilera CM, Olza J, Gil A. Genetic susceptibility to obesity and metabolic syndrome in childhood. Nutr Hosp. 2013 Sep;28 Suppl 5:44-55.

37. Blencowe M, Karunanayake T, Wier J, Hsu N, Yang X. Network Modeling Approaches and Applications to Unravelling Non-Alcoholic Fatty Liver Disease. Genes (Basel). 2019 Nov 24;10(12):966.

38. Liu LL, Liu L, Liu HH, Ren SS, Dou CY, Cheng PP, Wang CL, Wang LN, Chen XL, Zhang H, Chen MT. Levamisole suppresses adipogenesis of aplastic anaemia-derived bone marrow mesenchymal stem cells through ZFP36L1-PPARGC1B axis. J Cell Mol Med. 2018 Sep;22(9):4496-4506. 
39. Tarling E J , Clifford B L , Cheng J , et al. RNA-binding protein ZFP36L1 maintains posttranscriptional regulation of bile acid metabolism[J]. The Journal of Clinical Investigation, 2017, 127(10).

Figures

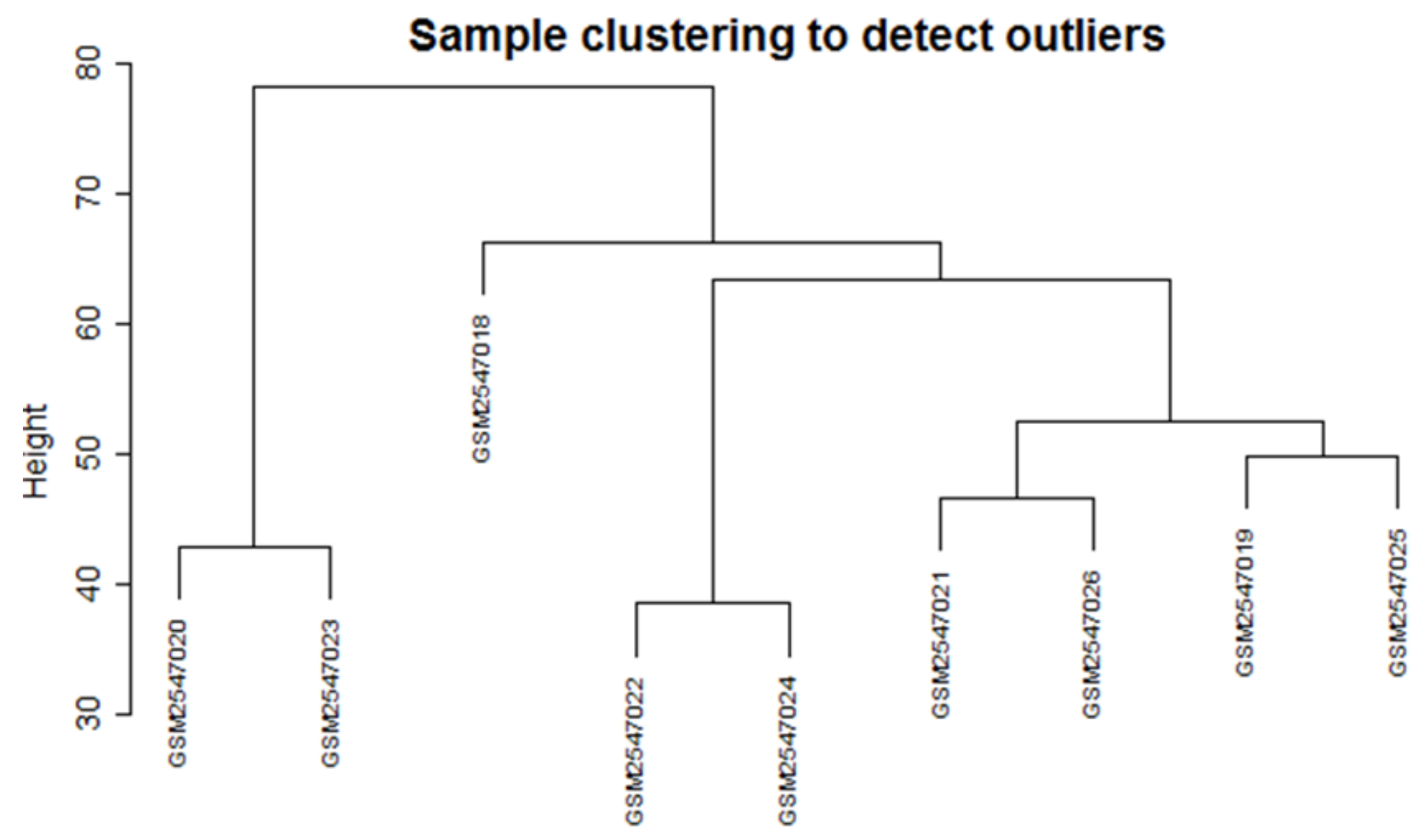

Figure 1

Cluster analysis of 9 NASH samples in GSE96971. 
(A) Scale independence

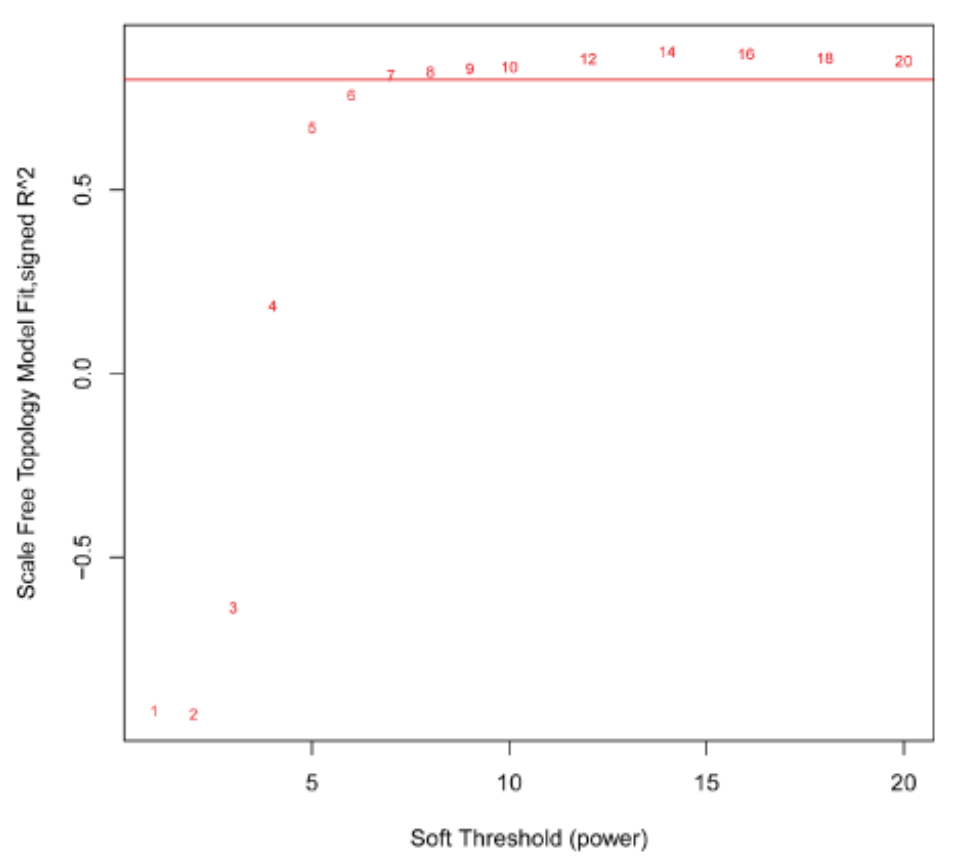

(B) Mean connectivity

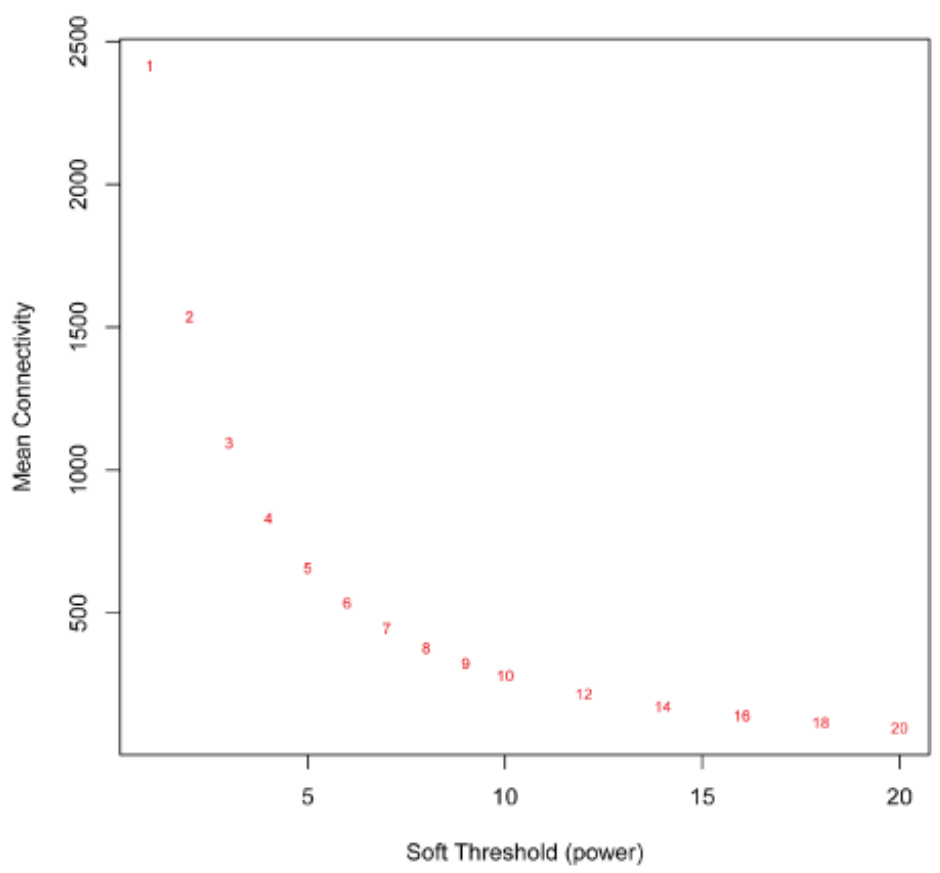

(C) Gene Dendrogram and module colors

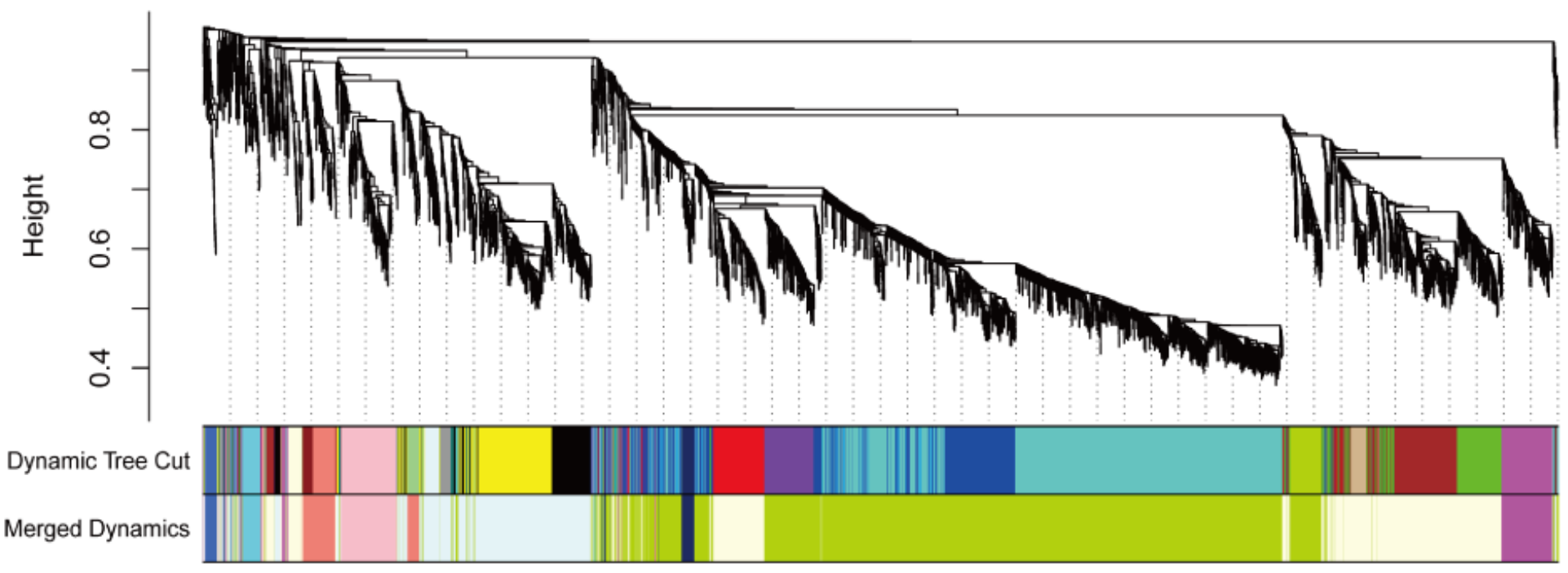

\section{Figure 2}

Co-expression module of NASH-related genes. (A) The effect of distinct values on scale independence of gene co-expression module. (B) The diversity control value is the normal association of the co-expression module. (C) Establish the co-expression module through the WGCNA method. 


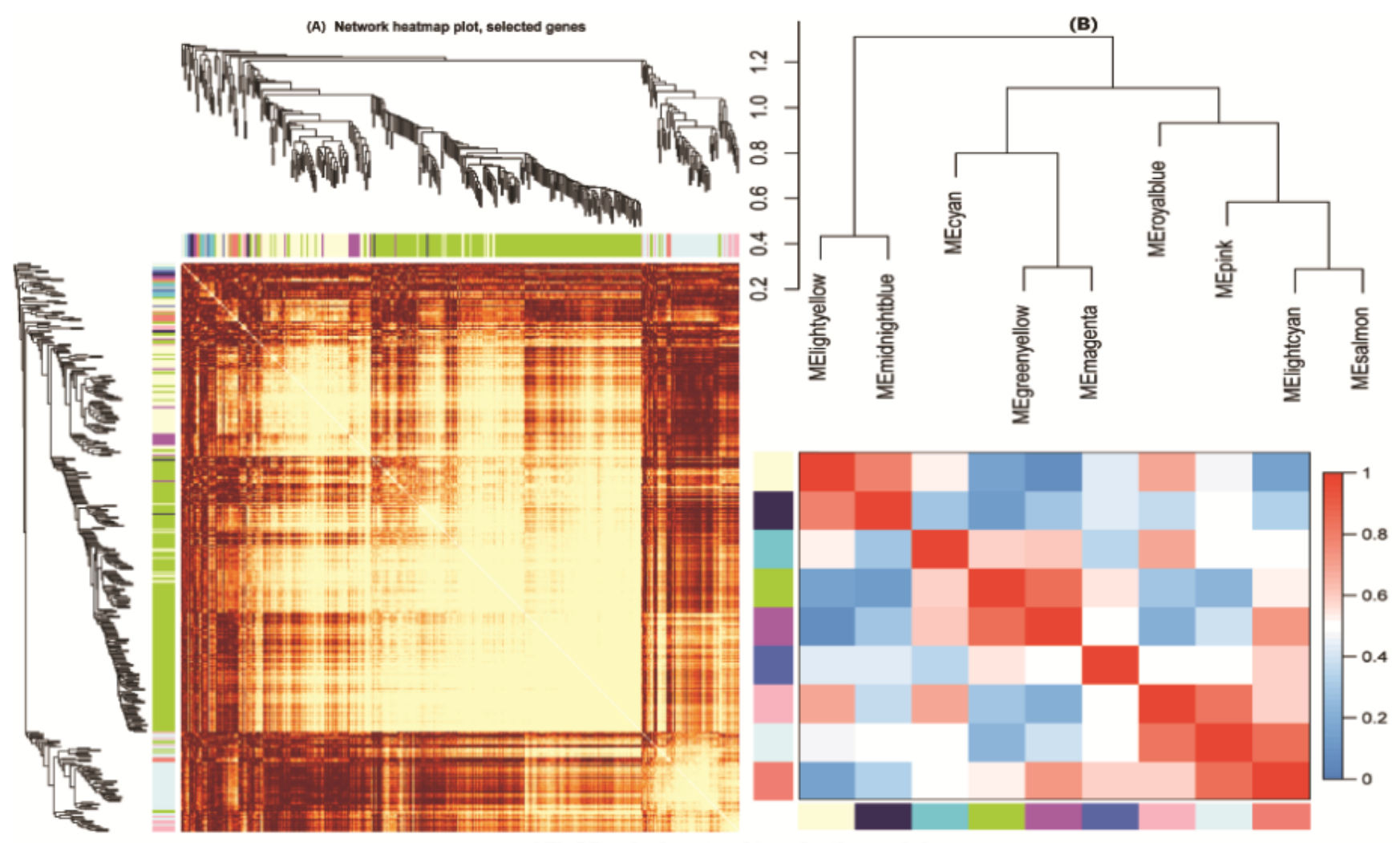

(C) Module-trait relationships

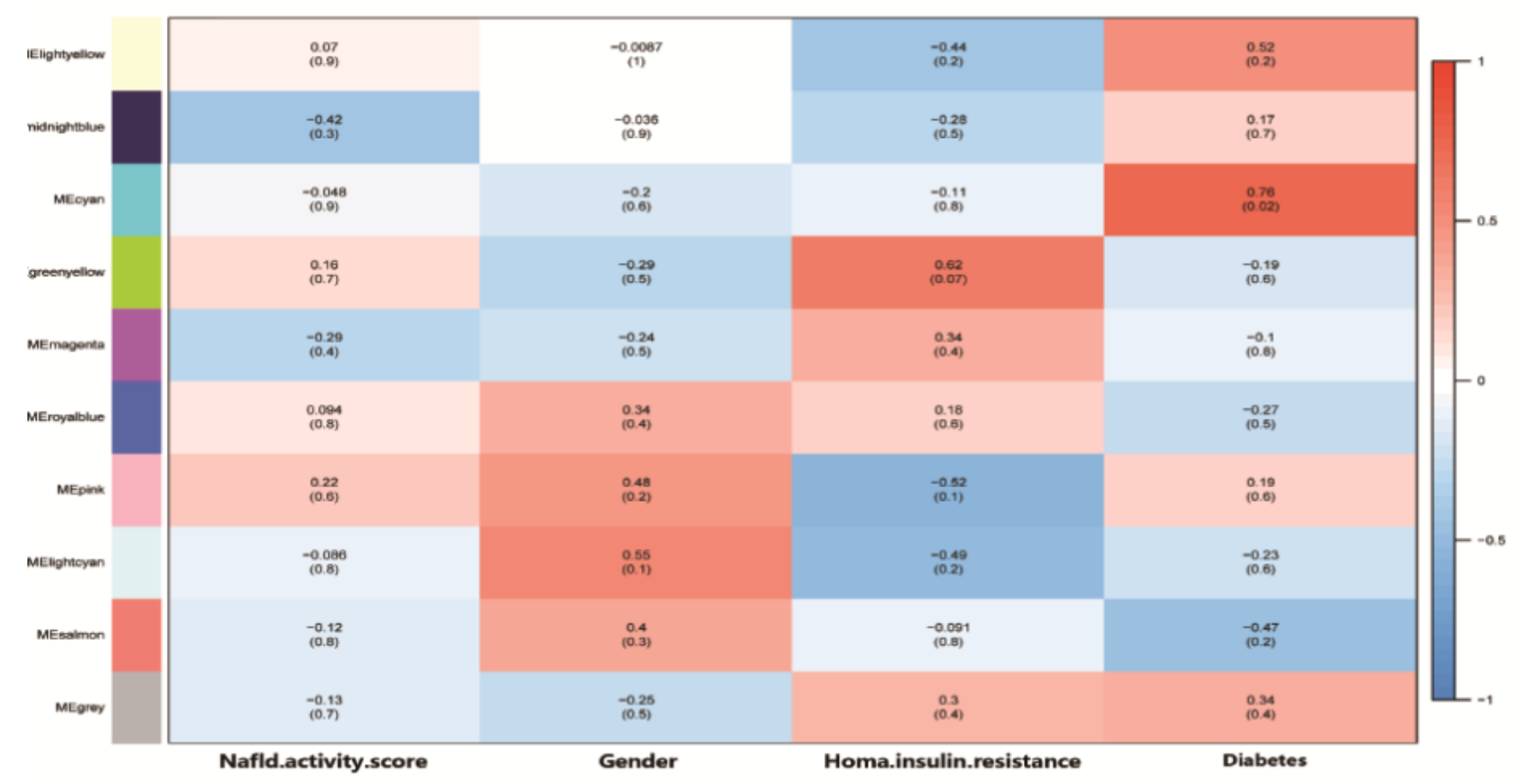

Figure 3

Modules analysis and identified. (A) Association analyses for co-expression modules. Distinctive modules are shown by different colors oriented on vertical or horizontal axis. The relevance and importance of quality is demonstrated through the brilliance of the yellow color. (B) Connectivity examination of eigengenes (you mean eight genes?) of distinctive modules. A total of 10 modules were 
constructed. Grey module cannot be clustered into remaining 9 modules. (C) Correlation between modules and NASH. The degree of correlation of module is shown by the red color.

\section{(A) Module-trait relationships}

\begin{tabular}{|c|c|c|c|c|c|}
\hline MEcyan & $\begin{array}{l}0.003 \\
0.77)\end{array}$ & $\begin{array}{l}0.08 \\
(20-28)\end{array}$ & $\begin{array}{l}0.006 \\
(0.7)\end{array}$ & $\begin{array}{c}0.073 \\
(0.7)\end{array}$ & $\begin{array}{l}0.033 \\
(0.8)\end{array}$ \\
\hline MEgreen & $\begin{array}{l}0.18 \\
(0.3)\end{array}$ & $\begin{array}{l}0.073 \\
(0.6)\end{array}$ & $\begin{array}{l}-0.2 \\
(0.2)\end{array}$ & $\begin{array}{l}-0.081 \\
(0.7)\end{array}$ & $\underset{\text { (1) }}{0.0033}$ \\
\hline greenyellow & $\begin{array}{l}0.76 \\
(90-09)\end{array}$ & $\begin{array}{l}0.25 \\
(0.1)\end{array}$ & $\begin{array}{l}0.33 \\
(0.04)\end{array}$ & $\begin{array}{l}0.37 \\
(0.02)\end{array}$ & $\begin{array}{l}0.27 \\
(0.09)\end{array}$ \\
\hline MEpink & $\begin{array}{c}0.74 \\
(2 e-08)\end{array}$ & $\begin{array}{l}0.048 \\
(0.8)\end{array}$ & $\begin{array}{l}0.23 \\
(0.1)\end{array}$ & $\begin{array}{l}0.28 \\
(0.1)\end{array}$ & $\begin{array}{l}0.22 \\
(0.2)\end{array}$ \\
\hline MEturquoise & $\begin{array}{l}-0.35 \\
(0.02)\end{array}$ & $\begin{array}{l}-0.18 \\
(0.3)\end{array}$ & $\begin{array}{l}-0.026 \\
(0.9)\end{array}$ & $\begin{array}{l}0.088 \\
(0.6)\end{array}$ & $\begin{array}{l}-0.12 \\
(0.4)\end{array}$ \\
\hline MEmagenta & $\begin{array}{l}-0.48 \\
(0.001)\end{array}$ & $\begin{array}{l}-0.014 \\
(0.9)\end{array}$ & $\begin{array}{l}-0.41 \\
(0.008)\end{array}$ & $\begin{array}{l}-0.36 \\
(0.02)\end{array}$ & $\begin{array}{l}-0.2 \\
(0.2)\end{array}$ \\
\hline MEtan & $\left(\begin{array}{l}-0.9 \\
(10-15)\end{array}\right.$ & $\begin{array}{l}-0.04 \\
(0.8)\end{array}$ & $\begin{array}{l}-0.38 \\
(0.011\end{array}$ & $\begin{array}{l}-0.44 \\
(0.004)\end{array}$ & $\begin{array}{l}-0.29 \\
(0.06)\end{array}$ \\
\hline MEblack & $\begin{array}{l}-0.47 \\
(0.002)\end{array}$ & $\begin{array}{l}-0.16 \\
(0.3)\end{array}$ & $\begin{array}{l}-0.3 \\
(0.06)\end{array}$ & $\begin{array}{l}-0.43 \\
(0.005)\end{array}$ & $\begin{array}{l}-0.12 \\
(0.4)\end{array}$ \\
\hline MEsalmon & $\begin{array}{l}-0.31 \\
(0.05)\end{array}$ & $\begin{array}{l}-0.47 \\
(0.002)\end{array}$ & $\begin{array}{l}-0.035 \\
(0.8)\end{array}$ & $\begin{array}{l}-0.2 \\
(0.2)\end{array}$ & $\begin{array}{l}-0.077 \\
(0.6)\end{array}$ \\
\hline MEgrey & $\begin{array}{l}0.043 \\
(0.8)\end{array}$ & $\begin{array}{l}-0.069 \\
(0.7)\end{array}$ & $\begin{array}{l}0.18 \\
(0.3)\end{array}$ & $\begin{array}{l}0.24 \\
(0.1)\end{array}$ & $\begin{array}{l}0.15 \\
(0.3)\end{array}$ \\
\hline
\end{tabular}

GSE96971

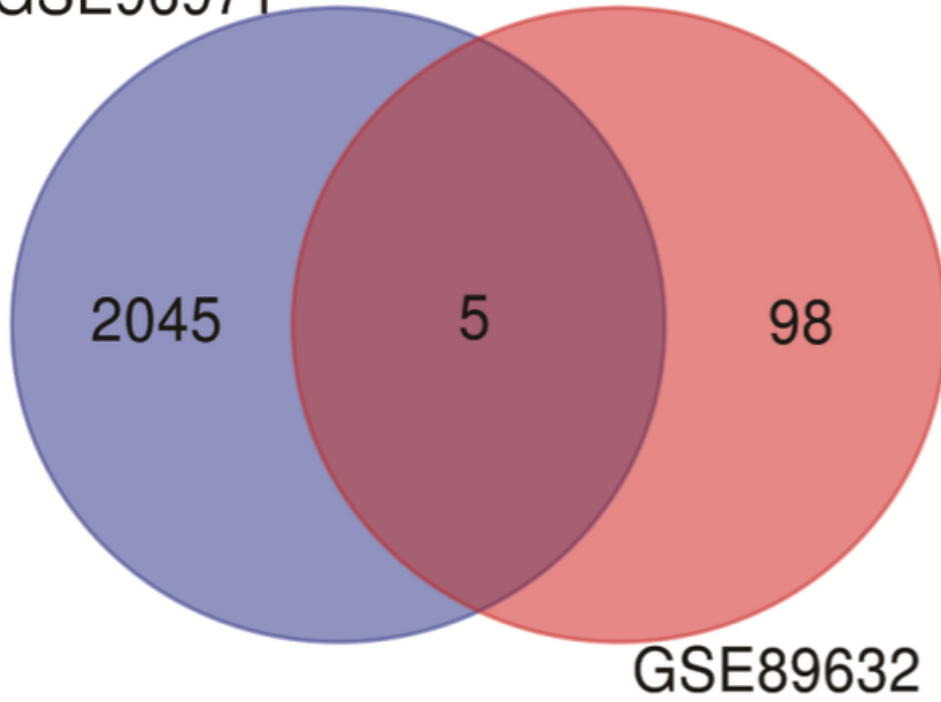

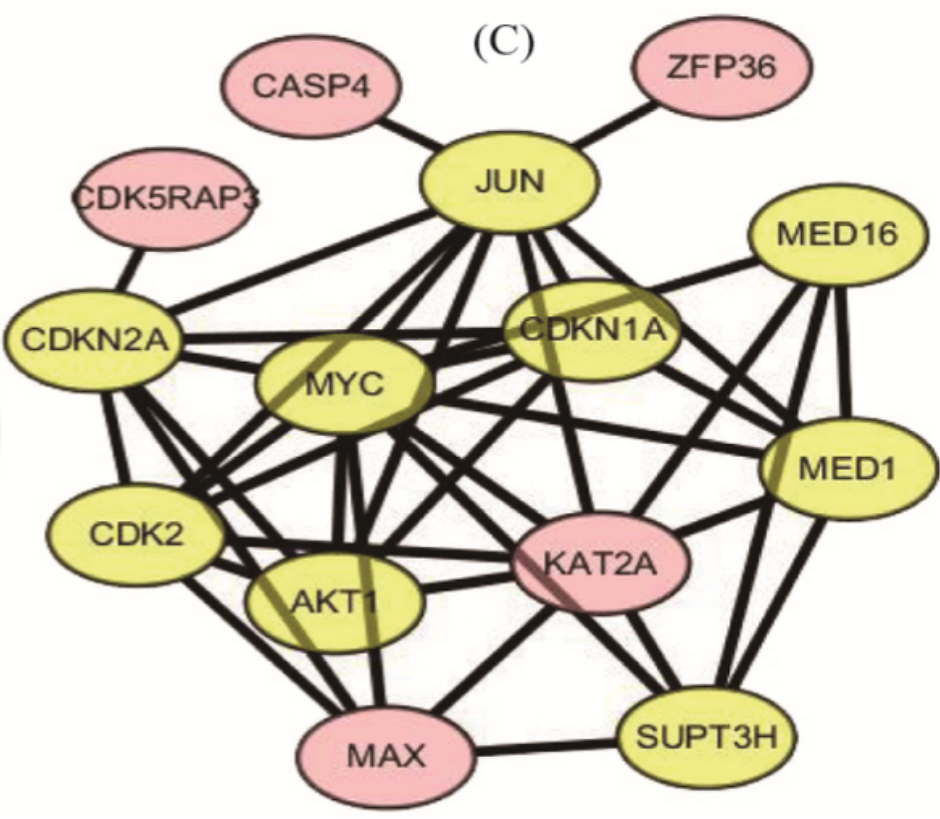

Figure 4

Hub genes are identified and verified. (A) Correlation between modules and insulin resistance genes in GSE89632 data set. (B) The Venn diagram of hub genes of GSE89632 and GSE96971 data sets were identified and verified) (C) Network analysis of the hub genes in yellow-green module. 


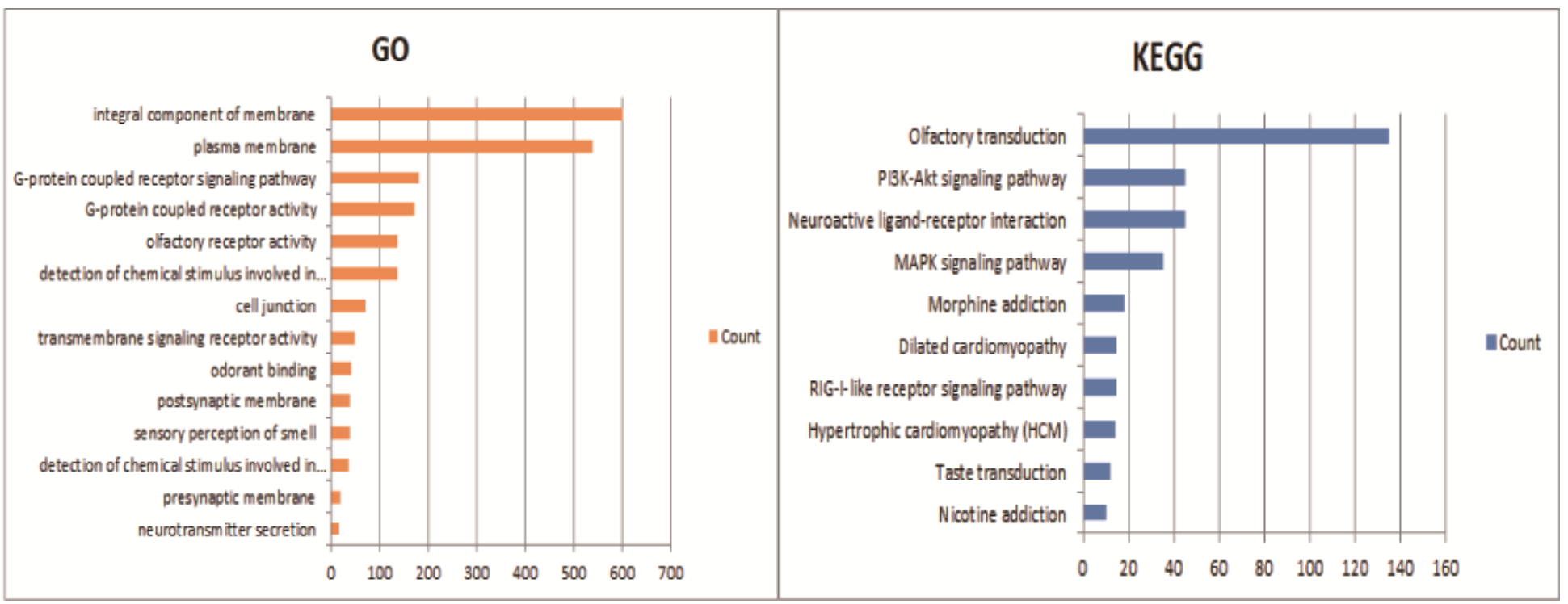

Figure 5

The top ten GO and KEGG terms of the greenish-yellow module in GSE96971 data set.
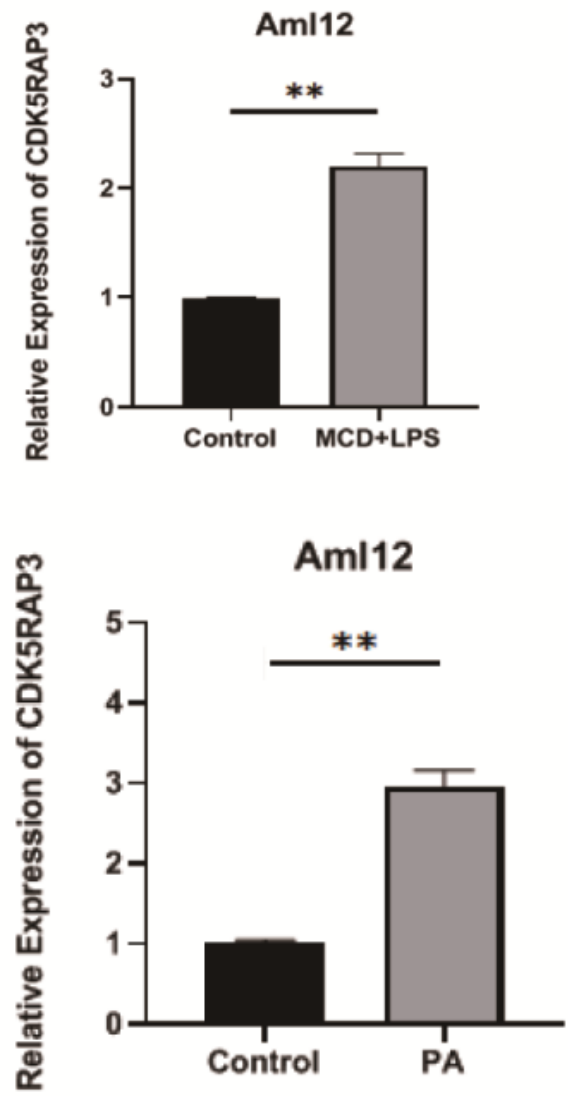
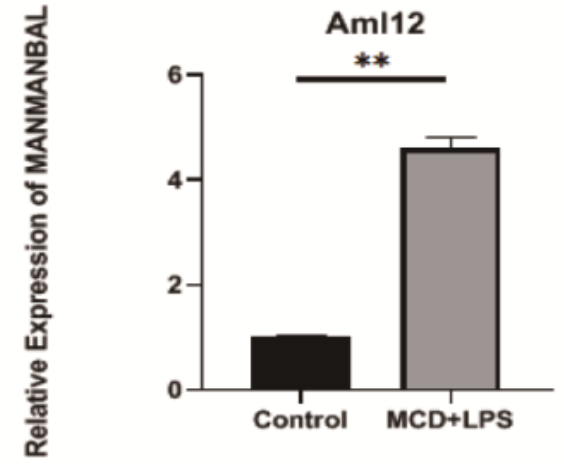



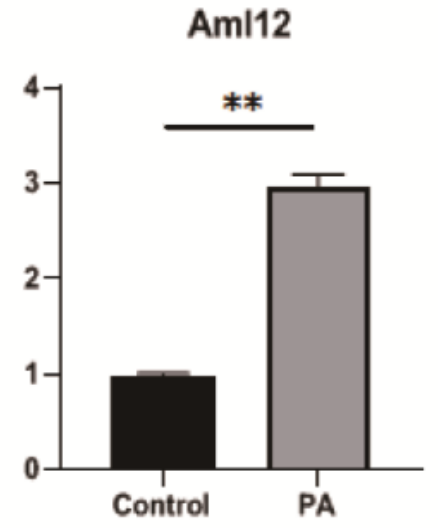

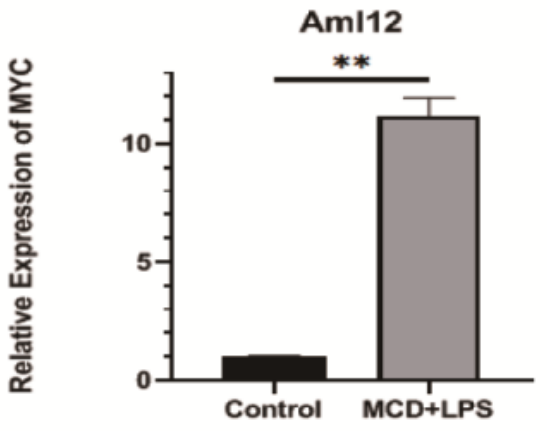

Aml12

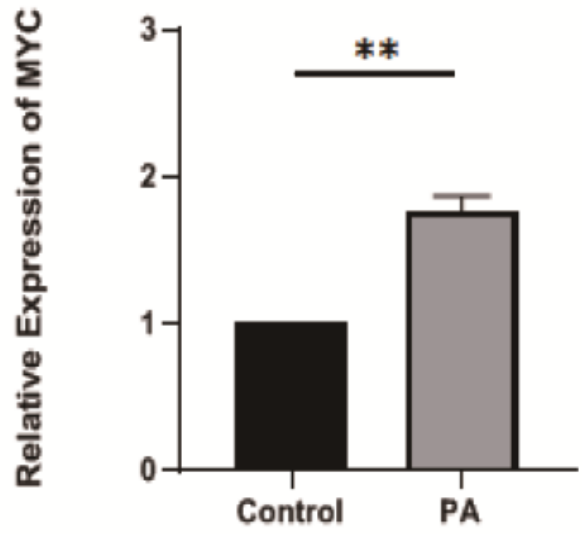

Figure 6

Relative expression levels of MANBAL, MYC and CDK5RAP3 mRNA between two NASH cell models and control group, ** means $P<0.01$. 
A
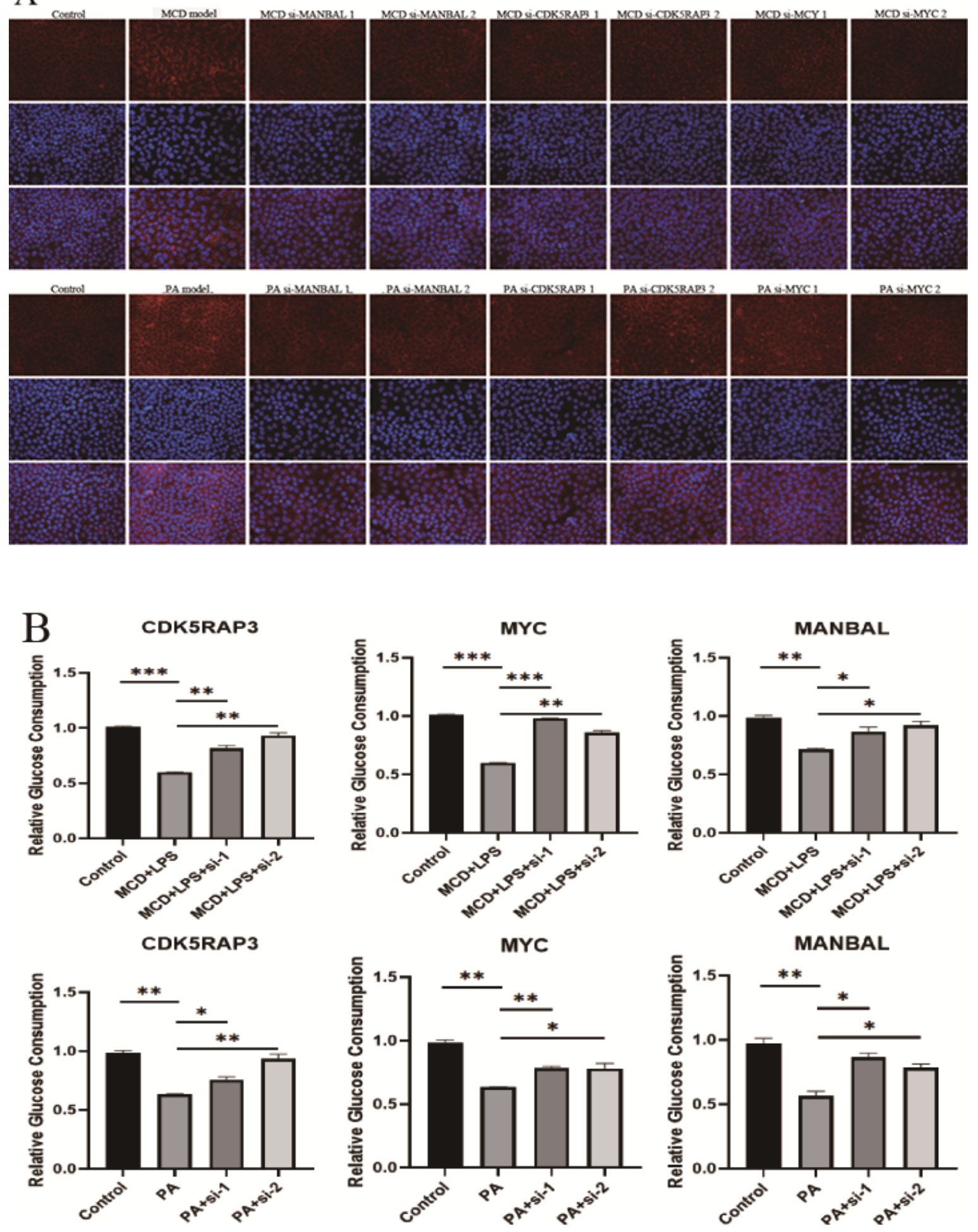

Figure 7

(A) The lipid deposition levels in the control group, two NASH cell model groups, and MANBAL/MYC/CDK5RAP3 knockout in two NASH cell model groups. (B) The glucose consumption levels in the control group, two NASH cell model groups, and MANBAL/MYC/CDK5RAP3 knockout in two NASH cell model groups. 